\title{
Intervención y puesta en valor de la Torre de Los Caballos Un nuevo espacio museístico dedicado a las torres vigía de la costa de Mazarrón
}

\author{
Pedro E. Collado Espejo
}

Universidad Politécnica de Cartagena, Cartagena, España, pedroe.collado@upct.es

\begin{abstract}
The Tower of Los Caballos is one of the three watchtowers built between the 15th and 16th centuries in Mazarrón (Murcia - Spain), in order to warn the population of eventual incursions by Berber pirates. The Tower is located on the coastline and is currently annexed to the Immaculate Conception Chapel. It has a square plan, two internal levels and a crenelated roof, built of stone and lime masonry. With the restoration and enhancement of the Tower, the original structures have been consolidated, by improving accessibility and internal roominess and its adaptation to the environment. Moreover, a museum space has been created with information panels and didactic materials devoted to "Watch towers and the history of the Miracle”, which enhances this Tower as an important tourist, cultural and leisure resource of Mazarrón.
\end{abstract}

Key words: tower, musealization, cultural tourism.

\section{Introducción}

La Torre de Los Caballos, en Bolnuevo, se ubica en plena línea de costa de la Región de Murcia, dejando al interior y a unos 5 kilómetros la población de Mazarrón (Comarca del Bajo Guadalentín, en el Sureste de la Región). Se trata de una de las tres torres defensivas costeras que fueron construidas entre los siglos XV y XVI, con la puesta en marcha de la explotación del alumbre, y que aún se conservan en este municipio; las otras dos son la Torre de Santa Isabel o de Las Cumbres, en el puerto de Mazarrón, y la Torre del Molinete, en pleno casco urbano de la ciudad. Estas torres vigía, declaradas Bien de Interés Cultural con categoría de Monumento (con la entrada en vigor de la Ley 16/1985 de Patrimonio Histórico Español), se construyeron para vigilar y poder dar aviso, para su defensa, a la población de la costa y el interior ante las frecuentes incursiones de piratas procedentes de la costa del Norte de África que

saqueaban el litoral murciano en aquella época, dificultando de esta manera la repoblación de estas tierras y la explotación de sus muchos recursos naturales.

Actualmente la Torre de Los Caballos, en Bolnuevo-Mazarrón, se encuentra anexa a la Ermita de la Purísima Concepción, formando un mismo conjunto aunque ambas con accesos independientes La intervención para la rehabilitación y puesta en valor de esta Torre defensiva y su nuevo uso como espacio museístico dedicado a las torres vigía de la costa de Mazarrón se engloba dentro de las actuaciones previstas en el "Plan Director de Infraestructuras, Accesibilidad y Equipamientos Turísticos de la Bahía de Mazarrón”, redactado por los arquitectos D. Rafael Pardo Prefasi y D. Severino Sánchez Sicilia y aprobado por el Consistorio mazarronero en 2003. Este Plan Director marcaba como uno de los principales 
objetivos a desarrollar en los años siguientes la planificación de diferentes actuaciones para la mejora, en todos los ámbitos, de la Bahía de Mazarrón con el fin de convertirla en un referente cultural y turístico de la Región de Murcia, prestando especial atención a todos los recursos históricos, sociales, arquitectónicos, naturales, paisajísticos y culturales que posee esta zona y a su potencialidad. En concreto, este Plan Director, en el apartado correspondiente al análisis y propuestas de actuación para mejorar y potenciar los numerosos "Recursos Históricos y Culturales” de la zona, detalla unos criterios y metodología de intervención que se han seguido en la rehabilitación y puesta en valor de la Torre de Los Caballos con el objetivo final de la creación de un nuevo espacio museístico dedicado a las torres vigía del litoral de Mazarrón potenciando así la Torre como recurso no sólo histórico y arquitectónico sino también cultural y turístico. Y todo ello prestando especial atención a la recuperación integral del Bien de Interés Cultural para su correcto mantenimiento y conservación.

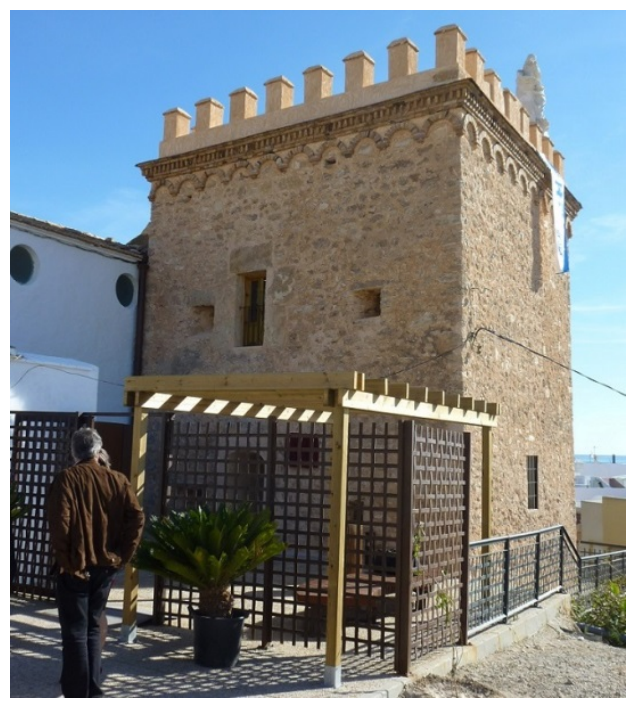

Fig. 1.- Torre de Los Caballos después de la intervención. (P.E. Collado)

\section{Breve descripción de la Torre}

La Torre de Los Caballos es una construcción militar, datada en el siglo XVI, de carácter defensivo y situada en alto sobre la playa de
Bolnuevo, para vigilar y alertar a la población ante posibles invasiones corsarias desde el mar. Es de planta cuadrada y está construida con muros de carga de mampostería en piedra caliza tomada con argamasa de cal y presenta dos niveles interiores, además de una cubierta con almenas.

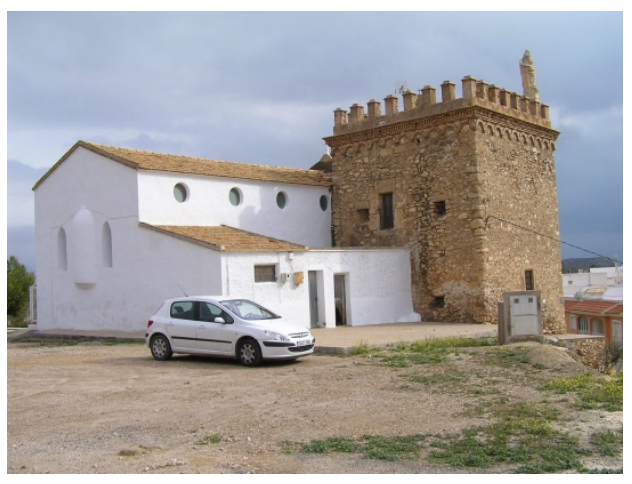

Fig. 2.- Imagen general de la Torre de Los Caballos y la ermita de la Purísima Concepción, antes de la restauración. (P.E. Collado)

La imagen actual de la Torre de Los Caballos se encuentra muy alterada respecto a lo que debió ser esta edificación militar en su origen. Actualmente, el acceso a esta construcción se hace por una única puerta situada en la planta baja. Sin embargo, originalmente el acceso debió ser por el nivel intermedio, en concreto donde en la actualidad se encuentra el hueco de mayor amplitud de la fachada principal (ahora una puerta balconera con barandilla metálica) y éste se realizaría a través de una escala para tener así una mayor seguridad y poder defenderse mejor de un posible ataque a la Torre.

Según está documentado, el actual acceso por el nivel inferior es fruto de una importante intervención realizada en la Torre durante el siglo XVIII. Posiblemente, en esta intervención también se debió vaciar el relleno interior que macizaba originalmente la Torre construyéndose el forjado intermedio y las actuales escaleras interiores de acceso a esta planta y a la cubierta. También se debió acondicionar la cubierta realizándose el actual remate decorativo de cornisa sobre arquillos ciegos de ladrillo y las almenas que coronan la Torre. 
Recibe el nombre de Torre de Los Caballos porque en origen tenía, entre sus construcciones anexas, unas caballerizas que hoy en día están desaparecidas y que ocuparían el lugar donde ahora se encuentra la Ermita de la Purísima Concepción. Estas caballerizas debían estar al servicio de los vigías y desaparecerían, como muy tarde, en el año 1946 que es cuando se construyó la actual Ermita reformando nuevamente la Torre. De esta intervención destaca la colocación de la figura de la Virgen que corona la terraza de la construcción.

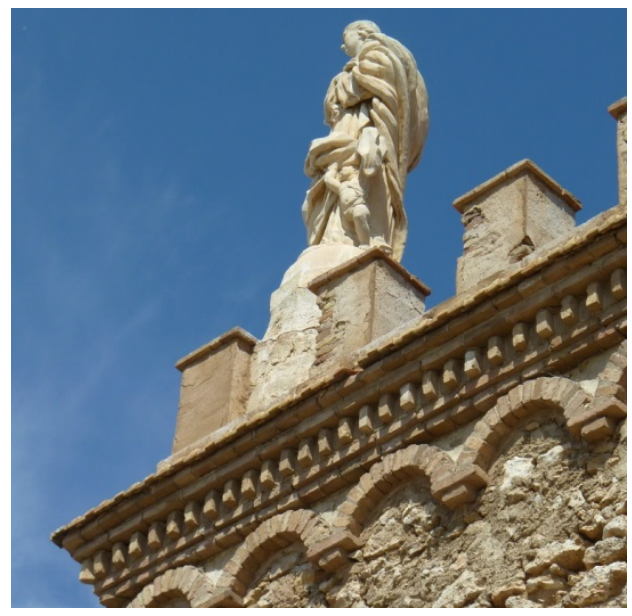

Fig. 3.- Imagen de la Virgen en la terraza de la Torre antes de la restauración. (P.E. Collado)

La construcción de la Ermita de la Purísima Concepción y la colocación de la imagen de la Virgen en la terraza de la Torre no es algo casual, sino que tiene mucho que ver con la historia de Mazarrón. Tal y como se describe en la exposición "El siglo del Milagro”, "En una de aquellas temidas incursiones corsarias, tuvo lugar un hecho trascendental conocido como El Milagro de la Virgen de la Purísima. Se cuenta que en la madrugada del 17 de noviembre de 1585, la tripulación de uno de aquellos barcos de piratas huyó por la intercesión de la Virgen, en forma de hermosa doncella rodeada de un gran resplandor. Sobre la arena de las playas quedaron las armas que en su fuga dejaron los corsarios y hasta una bandera, que aún conservamos, y que fueron halladas al amanecer por las gentes del lugar. Estas, al ir a agradecer a la Virgen el frustrado ataque, descubrieron en la ermita de la Concepción una lámpara de la que no paraba de manar aceite y que se hallaba sorprendentemente encendida, pese a que había sido vista aquella mañana seca y apagada; pero, sin duda, lo que más sobrecogió a aquellos vecinos fue el iluminado rostro de la Virgen del que brotaba un divino sudor y cuyo manto se encontraba mojado y con restos de arena. Los testigos de aquellos asombrosos hechos no tardaron en sacar conclusiones: la Virgen, a la que tantas veces habían rezado y solicitado protección, habría obrado el milagro de hacer huir a los corsarios”. Por tanto, la intervención que se está comentando también incidirá en la difusión de este hecho histórico.

En los últimos años la Torre no había sido objeto de restauración y antes de la intervención el acceso se realizaba a través de un pequeño patio, por detrás de la Ermita, que también comunicaba con la sacristía y con un pequeño aseo. No tenía un uso permanente, estando acondicionada para un alojamiento eventual, fundamentalmente para la época estival.

La Torre se encuentra en un entorno urbanizado, dentro del núcleo urbano de Bolnuevo, y la apertura de calles de los últimos años había abierto un importante desnivel en su lado sur, sujeto por una importante estructura de contención de tierras. Aunque por su elevación en origen contaría con importantes vistas sobre el frente marítimo, las nuevas edificaciones han ocultado la perspectiva.

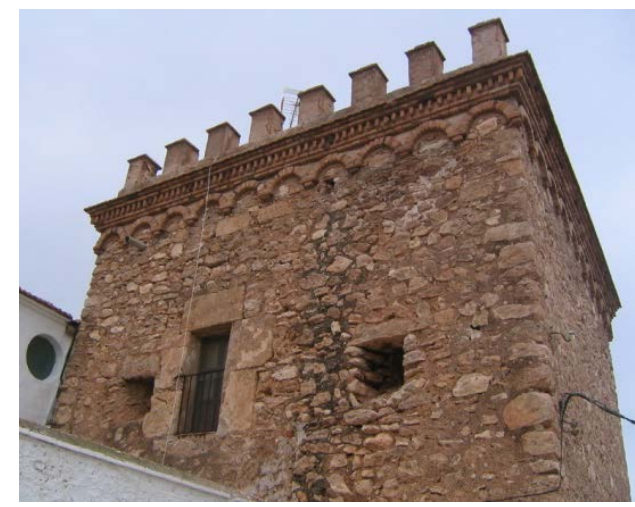

Fig. 4.- Detalle del estado de conservación de la Torre antes de la restauración. El actual balcón debió ser el acceso original. (P.E. Collado) 
En general, el estado de conservación de la edificación era relativamente bueno, ya que se encontraba en uso. No obstante requería actuaciones de mejora, fundamentalmente en cuanto a la limpieza general de los paramentos, restitución de las pequeñas lagunas de material pétreos en fachadas, tratamiento de las numerosas humedades por capilaridad en muros y filtraciones de cubierta, adaptación de las instalaciones de iluminación y electricidad, reparación del acceso a la cubierta, sustitución de los pavimentos interiores y de la terraza, restauración de las almenas, restauración de las carpinterías... Pero fundamentalmente la actuación debía centrarse en devolver el estado interior original a la Torre, sin las particiones del uso esporádico que se le estaba dando como apartamento, y acondicionar la edificación para albergar el nuevo espacio museístico dedicado a dar a conocer las torres vigía y toda la historia que hay alrededor de ellas.

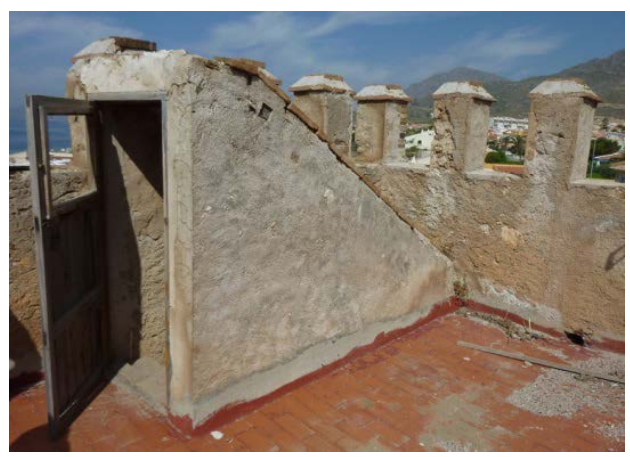

Fig. 5.- Detalle del estado de conservación del acceso a la cubierta antes de la restauración. (P.E. Collado)

\section{Objetivos de la actuación}

Las actuaciones proyectadas contemplaban tanto la restauración integral de la Torre de Los Caballos para albergar el nuevo uso museístico como el tratamiento, acorde con el futuro uso, de su entorno inmediato.

Se plantearon cuatro objetivos básicos. En primer lugar, se debía restaurar y consolidar la edificación, pero teniendo muy en cuenta que se interviene en un Bien de Interés Cultural con categoría de Monumento; por tanto, la metodología de conocimiento y los criterios de actuación debían ser absolutamente respetuosos con el carácter histórico, arquitectónico y patrimonial que atesora la Torre, limitándose a procesos básicos de limpieza, consolidación y conservación intentando, en todo momento, diferenciar, aunque de manera sutil, los materiales nuevos a colocar respecto de los originales, para evitar caer en el llamado falso histórico. En este caso, una de las actuaciones más importantes consistía en la eliminación de la tabiquería interior que convertía la Torre en un apartamento con un uso esporádico. El edificio, una vez restaurado y convenientemente acondicionado, debía albergar un nuevo espacio museístico por lo que era muy importante conseguir dos salas diáfanas, una por cada planta del inmueble, que permitieran una mejor adaptación como local expositivo. En segundo lugar, se buscaba una mejora de las condiciones de accesibilidad al inmueble. En este caso, a pesar de ser un objetivo derivado de la Ley Regional de Accesibilidad, por las características constructivas de la Torre, no resultaba posible garantizar la accesibilidad total al interior de la construcción sin alterar gravemente su configuración arquitectónica original (protegida por su carácter de Bien de Interés Cultural). No obstante, la actuación se encaminó a garantizar el acceso de todas las personas al menos a la planta baja de la Torre y, en cualquier caso, a los puntos donde se instalarán finalmente los carteles y puntos de información e interpretación de la intervención.

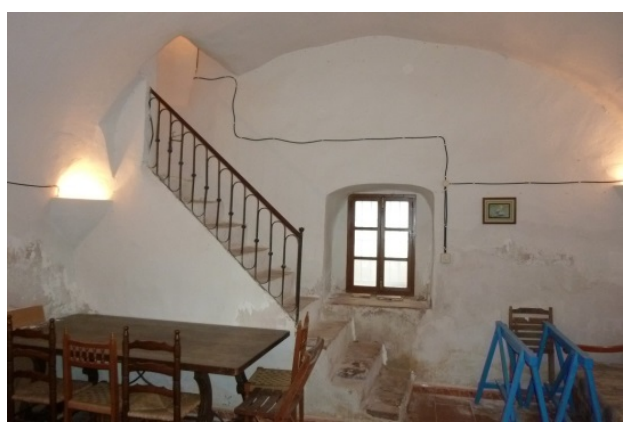

Fig. 6.- Planta baja interior de la Torre antes de la restauración. (P.E. Collado) 
En tercer lugar, se buscaba el acondicionamiento del entorno inmediato de la edificación histórica al objeto de mejorar su imagen y sus condiciones como espacio abierto de acogida de visitantes. Sobre este espacio, que queda delimitado delante del acceso principal de la Torre, se han centrado las actuaciones para la mejora de accesibilidad y las diferentes acciones informativas y didácticas proyectadas, pero además, se ha creado una pequeña zona de estancia y descanso de visitantes, con un mobiliario urbano compuesto por un banco, papelera, cartelería y una pérgola de madera diseñados acorde con el entorno histórico, arquitectónico y cultural que conforman la Torre y la Ermita.

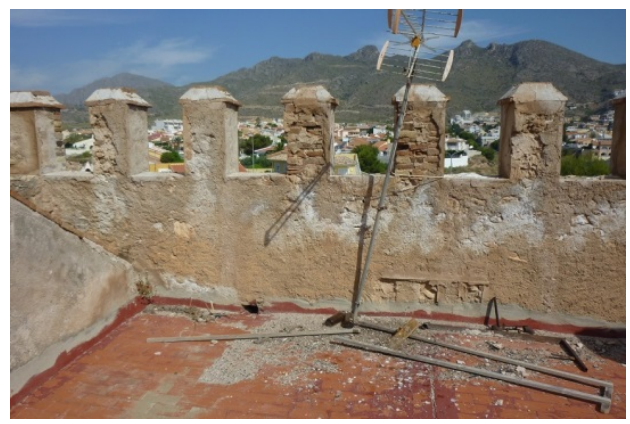

Fig. 7.- Detalle del estado de conservación de las almenas antes de la restauración. (P.E. Collado)

En cuarto lugar, se buscaba convertir la Torre de Los Caballos en un punto de información turístico-cultural, dotando al inmueble con contenidos no sólo específicos del propio monumento, sino generales del papel de las torres vigía en la historia del municipio de Mazarrón. Se pretende relacionar entre sí la información de las tres torres defensivas del municipio (la Torre del Molinete, la Torre de Santa Isabel o de Las Cumbres y la propia Torre de Los Caballos), como una actuación temática conjunta, creando una ruta turístico-cultural, que se podría denominar "Ruta de las torres vigía", extensible a otros municipios de la Región de Murcia e incluso a otras comunidades autónomas. Un contenido común para esta actuación informativa e interpretativa sería la comentada anteriormente historia del Milagro de la Purísima, relacionando también este acontecimiento, o más bien creencia popular, con las historias de la presencia de incursiones de piratas berberiscos en el litoral del municipio de Mazarrón.

\section{Intervención en la Torre de Los Caballos}

En primer lugar se procedió a la limpieza general de la edificación y su entorno inmediato, con extracción del mobiliario que aún quedaba en su interior y la eliminación completa de la tabiquería, en las dos plantas, para dejar los niveles exentos y poder, más adelante, adaptar la Torre como local expositivo. En las fachadas se procedió a la eliminación de las pintadas existentes con el uso de proyección de agua destilada a presión controlada y cepillado manual con cepillo de celdas suaves. Las pequeñas grietas de los muros de mampostería fueron inyectadas con lechada de cal y cosidas con varillas de fibra de vidrio, consiguiendo la continuidad y estabilidad estructural de la fábrica. En pequeñas zonas que presentaban lagunas y carencias de material, se realizó la reintegración con material pétreo de distinta tonalidad de la existente, aunque sin destacarse en exceso, y mortero de cal coloreado para su entonación con el mortero existente, en una intervención que perseguía la diferenciación sutil entre materiales originales y nuevos pero con un marcando interés de recomposición integradora, además de aplicar un tratamiento hidrofugante como protección final (probado previamente para que no afectase a la textura y tonalidad de los paramentos originales de mampostería y que no produjese brillos). Para el tratamiento de las molduras de ladrillo visto se procedió el mismo criterio.

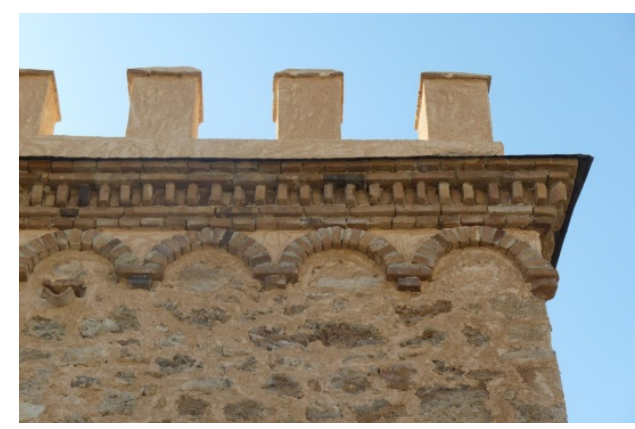

Fig. 8.- Detalle de las almenas y molduras de ladrillo ya restauradas. (P.E. Collado) 
Además, la cubierta presentaba un pavimento cerámico que no era el original y que se encontraba muy deteriorado por lo que se eliminó. Para evitar filtraciones, se rehicieron los faldones de cubierta y se impermeabilizó, colocándose un nuevo pavimento de baldosa de granito. Las almenas, que presentaban pérdidas de revoco fueron restauradas con mortero pétreo entonado con el resto de las fábricas. Para el acceso directo a la cubierta se eliminó la caseta de fábrica de ladrillo, muy deteriorada, y se colocó un nuevo casetón de acero corten

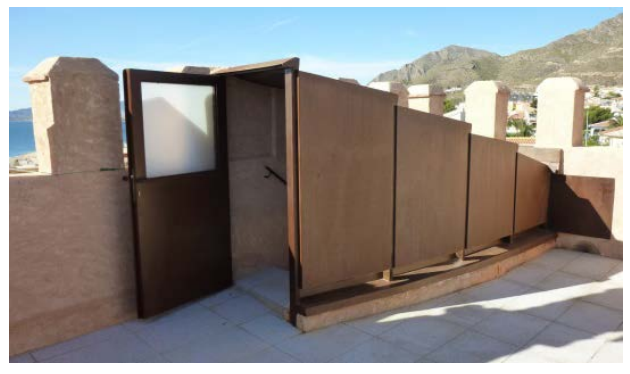

Fig. 9.- Detalle del casetón de acceso a la cubierta. (P.E. Collado)

Las intervenciones interiores se centraron en la eliminación de las humedades por capilaridad de los muros en planta baja mediante un forjado sanitario ventilado y enlucido con mortero y pintura transpirable. Los pavimentos cerámicos, muy deteriorados y con un aspecto relativamente moderno, se cambiaron por baldosas también cerámicas pero con aspecto más acorde al entorno histórico de la Torre. Las carpinterías eran producto de la reforma llevada a cabo anteriormente para acondicionar la edificación como apartamento por lo que se cambiaron por nuevas carpinterías de madera y herrajes de aspecto más de edificación patrimonial. En cuanto a las instalaciones de electricidad, agua y saneamiento se adecuaron todas a la normativa vigente pero teniendo muy en cuenta, especialmente con la instalación eléctrica y de iluminación, que el interior de la Torre tenía que albergar un nuevo espacio expositivo; se colocaron carriles de electrificación y luminarias en función a la posterior ubicación de los paneles informativos, carteles divulgativos, mobiliario expositivo y mesas de trabajo que formarían parte de la musealización proyectada para convertir la Torre en un nuevo referente cultural de Mazarrón. Actualmente, la musealización se ha visto impulsada con la oferta de visitas teatralizadas en las que los visitantes interactúan con un "pirata" que les enseña la Torre y explica cómo era la defensa de las costas, para terminar con unos juegos en los que los visitantes ponen en práctica todo lo aprendido.

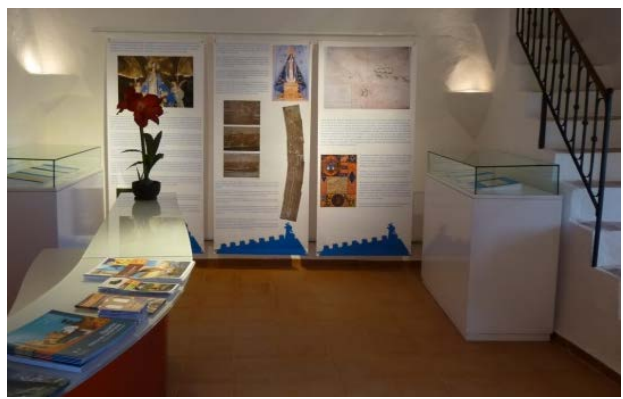

Fig. 10.- Plata baja acondicionada con paneles expositivos. (P.E. Collado)

\section{Intervención en el entorno de la Torre}

En cuanto a la intervención en el entorno próximo a la Torre se tuvo muy en cuenta que inicialmente la Torre y la Ermita formaban un único conjunto funcional, ya que ambas propiedades, una del Ayuntamiento de Mazarrón (la Torre) y otra del Obispado de Cartagena (la Ermita), no estaban delimitadas sino que compartían un acceso a través de un patio, donde también se encontraban un pequeño aseo, un trastero, una pequeña cocina al aire libre y una zona para acopio de material diverso. A este patio se accedía a través de una puerta metálica $y$, una vez en el patio se podía entrar bien a la Torre, por la puerta de planta baja, bien a la Ermita, a través de una puerta que daba acceso directo a la sacristía. Con esta intervención se pretendía dar respuesta al objetivo de permitir la visita de la Torre pero sin interferir en el normal uso de la Ermita por lo que el patio común debía ser reestructurado estableciendo accesos independientes para cada edificación. Además, estos nuevos accesos debían garantizar la seguridad anti-intrusión tanto de la Torre como de la Ermita y había que dignificar el aspecto de la visión de las fachadas lateral y trasera de la 
Ermita y la fachada de acceso principal de la Torre. En cuanto al pequeño aseo que había en el patio y que era principalmente para uso de la Ermita, la instalación debía mantenerse pero integrándola en la nueva solución de esta explanada de acceso a la Torre y sin que se destacase.

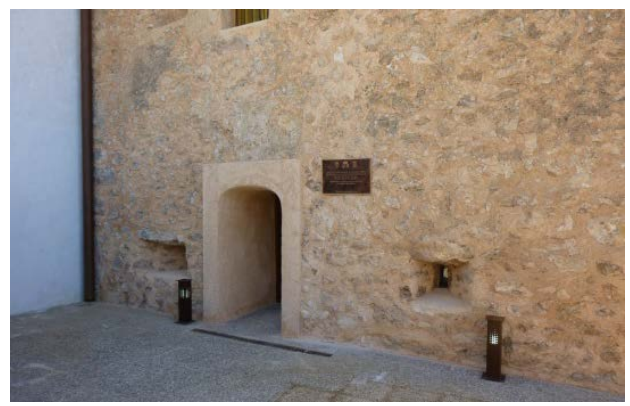

Fig. 11.- Acceso a la Torre de Los Caballos después de la restauración. (P.E. Collado)

Para cumplir con estos objetivos, la solución ha consistido en eliminar el cerramiento de ladrillo que configuraba el patio existente, permitiendo de esta manera una visión más directa y completa de la Torre y evitando que el acceso a la edificación histórica se produzca a través de un patio de pequeñas dimensiones y con un aspecto poco cuidado. Se eliminaron algunas de las construcciones auxiliares del patio, como el trastero y la pequeña cocina al aire libre, y se modificó el aseo. Con estas sencillas actuaciones la Torre adquiere ahora una presencia más exenta, estando adosada sólo al lateral de la Ermita pero mostrando la totalidad de la fachada de acceso.

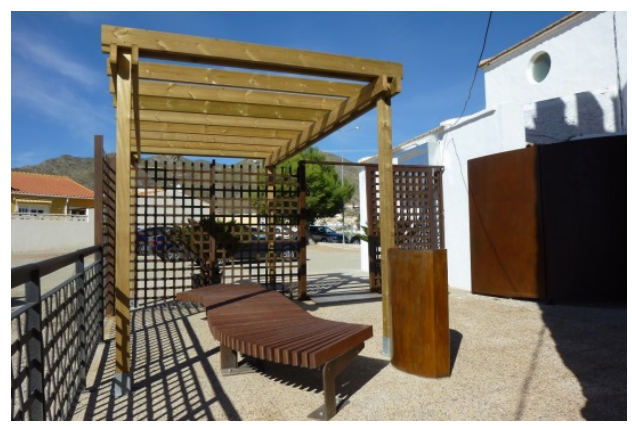

Fig. 12.- Zona estancial para control, acogida y descanso del visitante. (P.E. Collado)
Se ha creado una zona estancial, para el control de accesos, acogida y descanso de los visitantes, frente a la entrada a la Torre. Se trata de un espacio cerrado, para dotar de mayor seguridad a los accesos de la Torre y sacristía de la Ermita, en el que se ha colocado una decorativa reja con cancela. Se ha realizado una iluminación ornamental con balizas de diseño en acero corten $\mathrm{y}$, como nuevo mobiliario urbano, se han colocado un moderno banco de madera sin respaldo y formado una "s" en planta, una papelera en acero corten y una pérgola de madera. Para mejorar la imagen de esta zona estancial se ha colocado una pantalla curva, a modo de biombo, realizada en acero corten y con una altura de 2,50 metros que permite ocultar a la vista las construcciones menores que quedan adosadas al lateral de la Ermita, es decir, el aseo (que ahora está remodelado y sólo es accesible desde la sacristía) y un nuevo almacén, de reducidas dimensiones, que sustituye al viejo trastero que existía y que ahora sirve de cuarto de limpieza de la Torre.

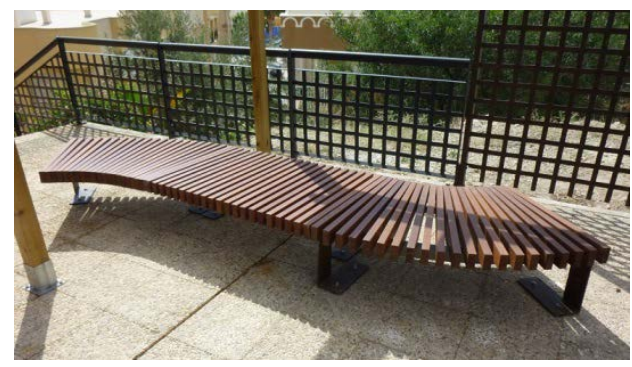

Fig. 13.- Detalle del banco de madera en la zona estancial. (P.E. Collado)

Así mismo, se ha protegido la caída hacia el talud existente en el lateral de la Torre con una barandilla de seguridad. El acceso a esta zona se realiza por la parte trasera de la Ermita, dejando un paso peatonal en la zona correspondiente al camarín de la Virgen.

Con todas las actuaciones que anteriormente se han descrito se ha conseguido dignificar la imagen del conjunto patrimonial, dotando de accesos independientes y seguros a la Torre y la Ermita, con lo que quedan perfectamente diferenciados los usos de ambas construcciones históricas pero manteniendo ambas propiedades plenamente su funcionalidad. 


\section{Conclusiones}

Las actuaciones desarrolladas en la Torre de Los Caballos han supuesto la restauración y puesta en valor de la antigua Torre vigía como un importante referente patrimonial de BolnuevoMazarrón. Antes de la intervención la Torre presentaba un aceptable estado de conservación pero con un uso, esporádico como apartamento, impropio de un Bien Cultural con categoría de monumento. Así mismo, se ha creado un nuevo espacio museístico dedicado a las torres vigía de la costa de Mazarrón potenciando la oferta cultural y de ocio de la ciudad.

La restauración ha permitido la consolidación de las estructuras originales, la mejora de las condiciones de accesibilidad y habitabilidad interior y la adecuación del entorno con la creación de una zona, vallada para controlar el acceso, acondicionada como lugar de estancia y descanso del visitante y con una iluminación ornamental y mobiliario urbano acorde al entorno histórico y patrimonial de la Torre de Los Caballos y la anexa Ermita de la Purísima Concepción. Además, se ha creado un espacio museístico, acondicionando las dos plantas interiores, con paneles expositivos y material didáctico dedicado a las "Torres vigía y la historia del Milagro”, potenciando esta Torre como un importante recurso turístico y cultural. Actualmente, la musealización se ha visto impulsada con la oferta de visitas teatralizadas en las que los visitantes interactúan con un “pirata” que les enseña la Torre y explica cómo era la defensa de las costas, para terminar con unos juegos en los que los visitantes ponen en práctica lo aprendido. Por tanto, la intervención y puesta en valor de la Torre de Los Caballos ha supuesto la recuperación de una edificación histórica y la creación de un nuevo espacio expositivo que complementa la oferta cultural y de ocio de Mazarrón.

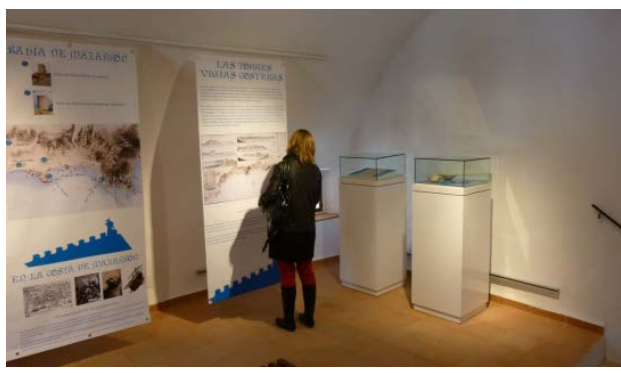

Fig. 14.- Detalle de la musealización de la planta primera. (P.E. Collado)

\section{Ficha técnica de la intervención}

Promotor/Propiedad: Consorcio Turístico de Mazarrón. Gerente: Pío Garrido Urbano.

Proyecto/Dirección de Obra: Rafael Pardo Prefasi, Severino Sánchez Sicilia, Inmaculada González Balibrea y Pedro-E. Collado Espejo. Empresa de restauración: Ingeniería Quipons SL Presupuesto de licitación: $116.000 €$

\section{Referencias}

Alonso S. (1990). Libro de los castillos y fortalezas de la Región de Murcia. Asociación Nacional de Amigos de los Castillos. Murcia.

Collado P.E., González I., Pardo R., Sánchez S. (2011). "Restauración de la Torre de Los Caballos en Bolnuevo-Mazarrón, para su puesta en valor como recurso turístico y cultural”. In XXII Jornadas de Patrimonio Cultural de la Región de Murcia. Ediciones Tres Fronteras. Murcia, pp. 213-221.

Gómez J.A., Martínez J.A. Munuera D. (2003). Castillos y fortificaciones de la Comarca de Cartagena desde la época púnica hasta nuestros días. Ligia Comunicación. Murcia.

Martínez J.A., Munuera D. (2009). Por tierra de Castillos. Guía de las fortificaciones medievales de la Región de Murcia y rutas por sus antiguos caminos. Ediciones Tres Fronteras. Murcia.

Munuera D. (2006). "Una historia singular: la costa del Reino de Murcia y el nacimiento de Mazarrón durante la etapa fronteriza (siglos XIII-XVI)”. In El Siglo del Milagro. Casas y Villa de los Alumbres de Almazarrón. Ligia Comunicación. Ayuntamiento de Mazarrón. pp. 22-39.

Pardo R., Sánchez S. (2003). Plan Director de Infraestructuras, Accesibilidad y Equipamientos Turísticos de la Bahía de Mazarrón. Ayuntamiento de Mazarrón. 